\title{
Profils discursifs de la combinatoire des verbes d'émotion en français et en arabe
}

\author{
Actancial Structures and Discursive Profiles of the Combinations \\ of Emotion Verbs in French and Arabic
}

\section{RÉSUMÉ}

Le travail se situe au niveau syntaxique et discursif : les emplois actifs des $\mathrm{V}$ affect en français et en arabe : deux verbes d'émotion (énerver et son équivalent [Yaydaba] et deux verbes de sentiment (admirer et son équivalent [?aЯzaba]). Ces verbes sont étudiés dans la perspective des dynamiques informationnelles au sein de la phrase (Van Valin et LaPolla, 1997). L'étude est fondée sur le corpus journalistique français EmoBase (projet Emolex $120 \mathrm{M}$ de mots) et sur le corpus journalistique ArabiCorpus (130 M de mots).

Mots-clés : combinatoire, affect, traduction, analyse contrastive, analyse actantielle, corpus

\section{ABSTRACT}

The work is at the syntactic and discursive level: the active form of the verbs affects in French and Arabic: two verbs of emotion (to rage and its equivalent [Yaydaba] and two verbs of feeling (to admire and its equivalent [?aSzaba]). These are studied from the perspective of information dynamics within the sentence (Van Valin \& LaPolla, 1997). The study is based on the French journalistic corpus EmoBase (Emolex 120M word project) and on the journalistic corpus ArabiCorpus (130M words). Keywords: combination, affect, translation, contrastive analysis, functional analysis, corpus

\section{Introduction}

Certains travaux cherchant à circonscrire la classe des verbes d'émotion (V_émotion) partent de la syntaxe pour étudier le sens (par exemple Gross, 1975; Harris, 1988). D'autres partent, à l'inverse, d'ensembles sémantiquement similaires de verbes afin d'aborder leurs propriétés syntaxiques (Mathieu, 2000; Buvet, Girardin, Gross, \& Groud 2005)‥ En revanche, la composante discursive est,

1 Zouaidi (2018) étudie la combinatoire des V_affect en français et en arabe d'un point de vue syntaxique et lexical. L'articulation de l'étude des structures actancielles (interface syntaxique)

Safa Zouaidi, Language Laboratory and Automatic Processing, Faculty of Arts and Humanities, University of Sfax, Airport Road Km 0.5, NO 1169, 3029 Sfax / Le Laboratoire de Linguistique et Didactique des Langues, Etrangères et Maternelles, Université Grenoble Alpes, Batiment, Stendhal,1086-1366 Avenue Centrale, 38400 Saint-Martin-d'Hères, szouaidi@yahoo.com, https://orcid.org/0000-0003-1366-6808 
à notre connaissance, prise peu en compte dans les recherches linguistiques réalisées sur ce lexique (Novakova, Goossens, \& Grossmann, 2013 et Novakova, Grossmann, \& Goossens, 2013). Dans cette contribution, nous nous intéresserons au profil discursif de la combinatoire des $\mathrm{V}$ émotion en fraçais et en arabe. Nous supposons que ce profil varie selon les choix discursifs du locuteur, en fonction de la présence ou de l'absence des actants syntaxiques (Asy) et des rôles sémantiques (Asé) que ces verbes véhiculent, et ce dans les deux langues comparées.

Après une présentation du cadre théorique (section 1.1), des corpus sur lesquels nous fondons cette étude (section 1.2) et de la méthodologie abordée dans ce travail (1.3), nous proposerons une étude qualitative sur le profil discursif des V_émotion ${ }^{2}$ (section 2.1) et des V_sentiment (section 2.2) dans la diathèse active ${ }^{3}$, et ce, dans les deux langues.

\section{Cadre théorique, corpus et méthodologie}

\subsection{Cadre théorique}

Nous choisissons de porter notre étude sur les combinaisons verbales (Bolly, 2010, 2011). Nous utilisons le mot «collocation » dans le sens de Hausmann (1989) dans la mesure où elle constitue la combinaison phraséologique binaire caractéristique de deux mots : base et collocatif (p. 1010). La collocation en arabe est traitée jusque là, à notre connaissance, autour du couple anglais-arabe sous un angle strictement traductologique (anglais-arabe) et lexicographique (El-Hasan, 1982; Abu-Ssaydeh, 2001; Ghazala, 2007).

De là, cette étude repose sur les différentes théories fonctionnelle (Halliday \& Hasan, 1976; Van Valin \& LaPolla, 1997) qui distinguent entre la valence abordée sous un angle syntaxique et la valence traitée sémantiquement des verbes, et insère systématiquement la dimension discursive à l'étude de leur fonctionnement syntaxique et sémantique (cf. Novakova, 2013). Les constructions syntaxiques constituent le moyen qui permet l'expression des significations sémantiques $\mathrm{du}$ locuteur. Nous visons ainsi à nous focaliser sur les visées communicatives de ce dernier en vue de démontrer le lien étroit entre les traits syntaxiques (les structures actancielles) des V_émotion étonner/[?adha]a] et énerver/[Sayḍaba] et des V_sentiment admirer/[?ązaba] et envier/[hasada], et les visées discursives du locuteur.

aux objectifs discursifs du locuteur (interface discursive) permettrait, à notre sens, d'aborder cette thématique dans une perspective fonctionnelle.

2 Les V_émotion : étiquette générique divisée en : V_émotion (causés, ponctuels et réactifs) vs V_sentiment (interpersonnels et duratif)

${ }^{3}$ Notre objectif est non pas de faire une simple description des profils syntaxico-discurcifs des verbes d'affect dans le cadre des trois diathèses (active, passive et pronominale), mais d'en proposer une analyse exhaustive fine dans la diathèse active. Des travaux futurs sur ces verbes au sein des emplois passifs et pronominaux pourront compléter cette étude. 


\subsection{Corpus}

Notre étude s'appuie sur deux corpus comparables et équilibrés (journaux quotidiens de presse écrite) $)^{4}$. Pour le français, nous nous basons sur l'interface EmoBase issue d'Emolex $\mathrm{ANR} / \mathrm{DFG}^{5}$. Pour l'arabe, nous nous fondons sur l'interface ArabiCorpus ${ }^{6}$. Nos deux corpus comprennent au total environ 255 millions de mots. Nous ne nous intéressons pas ici à l'ensemble du champ de l'émotion, mais plus particulièrement sur les structures qui combine un $\mathrm{V}$ _émotion et une lexie qui accompagne le verbe en question. Les émotions étudiées ici sont : la surprise, la colère, la jalousie et l'admiration. Au total, nous avons obtenu 815 co-occurrences d'associations qui comportent les verbes-bases : étonner/[Padhafa], énerver/ [?aydaba], admirer/[?ązaba] et envier/[hasada].

\subsection{Méthodologie}

Notre méthodologie privilégie une approche contrastive à partir de la constitution de corpus comparables. La langue de départ sera le français, orientation qui se fonde sur les observations d'un déséquilibre entre les recherches faites sur français et celles sur l'arabe en ce qui concerne le lexique de l'émotion. Il ne s'agit pas, évidemment, d'appliquer de manière mécanique des outils d'analyse conçus parfois pour une autre langue. Toutefois, les travaux faits sur le français pourront, à notre sens, s'appliquer avec profit à l'arabe tout en observant systématiquement les particularités de chaque langue.

Nous abordons ainsi les similitudes et les différences interlinguistiques entre deux mots dits «équivalents » (Bouchaddakh, 2010, p. 3). Ainsi, du côté sémantique, il s'agit de proposer des équivalents de traduction qui sont des « quasiéquivalents ou des équivalents partiels » (p. 4). Du côté syntaxico-discursif, c'est une description qui cible les structures syntaxiques en lien avec les visées discursives des lexies françaises et de leurs équivalents en arabe.

La question posée est alors de savoir si, pour des affects supposés universels (la surprise ou la colère, par exemple), nous pouvons retrouver des configurations syntaxico-discursives communes ou différentes. Devant la vaste étendue du terrain des affects, il a fallu penser à restreindre les champs sémantiques sur lesquels nous nous penchons dans cette étude. Cette restriction s'est faite par une sélection réalisée selon des critères particuliers (la polarité positive (admiration), négative (colère,

4 Devant l'absence, à notre connaissance, de corpus comparables arabe-français alignés sortis à la même époque, il nous a semblé indispensable de faire une étude sur de grands textes journalistiques contemporains dans les deux langues afin de parvenir à l'analyse linguistique (à double interface : syntaxique et discursive) la plus fine possible.

5 C'est un projet franco-allemand (2010-2013), cf. http://emolex.ugrenoble3.fr/emoBase/ (page consultée le 15 juillet 2019).

${ }_{6}$ L'interface ArabiCorpus est disponible en ligne, cf. http://arabicorpus.byu.edu/ (page consultée le 30 juin 2019). 
jalousie) ou neutre (surprise). La sélection des équivalents dans les deux langues est passée par une étape d'élimination en choisissant deux champs sémantiques en français et deux champs sémantiques en arabe, selon le degré d'adéquation aux critères précités, ce qui aboutit en tout à quatre champs sémantiques d'affect. Dans le tableau suivant, nous dressons la liste des champs sémantiques sélecionnés :

Tableau 1 : Les quatre champs étudiés dans les deux langues

\begin{tabular}{|c|c|c|}
\hline & Pour le français & Pour l'arabe \\
\hline \multirow{4}{*}{$\begin{array}{c}\text { Champs sémantiques } \\
\text { sélectionnés }\end{array}$} & Admiration & [?i९̧zābon] ${ }^{\mathrm{a}}$ \\
\hline & Colère & [raḍabon] $^{\mathrm{b}}$ \\
\hline & Jalousie & [yajraton] $^{\mathrm{c}}$ \\
\hline & Surprise & {$\left[\right.$ [mufāza?aton] ${ }^{\mathrm{d}}$} \\
\hline
\end{tabular}

a Le mot en arabe est : غإعجاب.

b Le mot en arabe est : غضب : غيجب.

c Le mot en arabe est : غيرة :

d Le mot en arabe est : مـ فاجةة:

Nous utiliserons les symboles X, Y, Z, qui viennent de Mel'čuk, Clas, et Polguère $(1999)^{7}$ et qui correspondent, respectivement, à l'expérienceur $(\mathrm{X})$, l'ob- $^{\prime}$ jet $(\mathrm{Y})$ et la cause $(\mathrm{Z})$. Dans cette partie, nous adopterons le système de codage conventionnel des actants de Novakova, Goossens, et Grossmann (2013, p. 33) :

Tableau 2 : Les actants : symboles utilisés

\begin{tabular}{|c|c|}
\hline Type d'acant & Codage \\
\hline Asy & $\mathrm{A} 1, \mathrm{~A} 2, \mathrm{~A} 3$ \\
\hline Asé & $\mathrm{X}$ expérienceur \\
& $\mathrm{Y}$ objet de l'émotion \\
& Z cause \\
& Zinst entité extérieure à la cause \\
& $\mathrm{P} / \mathrm{Z}$ propriété interne de la cause ${ }^{7}$ \\
& P/Y propriété de l'objet \\
& Y/Z fusion objet/cause \\
& $\mathrm{X} / \mathrm{Y}$ fusion expérienceur/objet \\
\hline
\end{tabular}

L'utilisation de ces symboles nous permet d'apporter une réponse à notre hypothèse selon laquelle le choix des actants, au sein de la phrase, pourrait avoir un effet sur la façon dont le locuteur organise son énoncé en vue de communiquer une information particulière.

7 Cf. aussi Mel'čuk, Clas, et Polguère (1999), qui font la distinction, dans une perspective lexicographique, entre trois niveaux d'organisation des actants. En revanche, notre contribution, est différente de cette approche lexicographique. 
Nous nous appuyons sur des dictionnaires bilingues français-arabe, arabefrançais dans la traduction des équivalents arabes. Pour les locuteurs non natifs, ceux-ci seront présentés en quatre lignes pour que leur lecture soit plus efficace :

1. L'original arabe (Orig. ar).

2. La translittération conformément à l'alphabet phonétique international (API) (Orig. translit.) ${ }^{8}$.

3. La traduction en français du mot original en arabe (tout en proposant de gloser les combinaisons des V_émotion citées ${ }^{9}$ selon les règles du Leipzig Glossing $^{10}$ (Trad. litt. $)^{11}$.

4. La traduction française des lexèmes arabes (Trad. fr.) ${ }^{12}$.

Dans la prochaine section, nous examinerons systématiquement le profil syntaxique et discursif des V_émotion (section 2.1) et des V_sentiment (section 2.2) au niveau phrastique (c'est-à-dire microtextuel) ${ }^{13}$.

\section{Profils discursifs des $\mathbf{V}$ _émotion au sein des emplois actifs}

L'étude dépasse le niveau syntagmatique pour atteindre le niveau phrastique ; le contexte sera ainsi pris en considération.

\subsection{Les $V_{-}$émotion}

Les verbes étonner et énerver, appartenant à la classe II des verbes psychologiques (Ruwet, 1994; Mathieu, 2000), marquent des émotions causées, réactives : étonner est de polarité neutre ; énerver, quant à lui, est de polarité négative. Leurs équivalents respectifs, [? $\underline{\text { ?adha }}$ a] et [?aydaba]), appartiennent à la forme $\mathrm{IV}^{14}$, qui est causative en arabe ${ }^{15}$.

La configuration prototypique des verbes étonner/[?adha]a] et énerver/[ৎayḍaba], en tant que deux émotions causées, est binaire : elle comprend l'Asé cause $(Z)$ et

8 Les locuteurs non natifs de cette langue pourront ainsi lire les lexèmes arabes.

9 Les phrases arabes sont souvent longues. Par conséquent, pour des raisons de place, seules les associations verbales d'affect ont été glosées.

${ }^{10}$ Cf. http://www.eva.mpg.de/lingua/resources/glossing-rules.php (page consultée le 30 juin 2019).

${ }^{11}$ Les exemples glosés permettent de donner des éclaircissements sur les significations sémantiques et les propriétés grammaticales des mots et des parties de mots, ce qui débouche sur une observation cohérente des traductions en mot à mot de la phrase arabe d'un point de vue syntaxique et sémantique.

${ }^{12}$ Les traductions proposées en bon français (ne sont pas littérales) permettent de mettre en œuvre la comparaison français-arabe.

${ }^{13}$ Le présent travail ne concerne pas le niveau d'analyse transphrastique (rôle du profil discursif pour l'argumentation de (et sur) les affects étudiés) (Sorba \& Novakova, 2013), ce qui pourrait être l'objet d'études futures.

${ }_{14}$ Pour plus de détails sur les dix formes verbales, en arabe, cf. Arbaoui (2010).

${ }^{15}$ Cf. aussi El Kassas (2005). 
l'Asé expérienceur $(\mathrm{X}): \mathrm{Z}$ étonnelénerve $\mathrm{X}$. Ces verbes peuvent figurer dans des structures à la fois monovalentes et bivalentes.

\subsubsection{Les structures monovalents}

Les V_émotion figurent dans des constructions où le deuxième actant sémantique (l'expérienceur $\mathrm{X}$ ) est absent. Le seul Asy exprimé correspond à la cause $(\mathrm{Z})$ de l'émotion :

(1)

Après l'opacité, ce sont les nylons, en superpositions cireuses comme des ailes d'insectes, ou des mailles translucides qui exposent le corps comme à travers un verre dépoli en teintes pastel, banane, pêche, ciel, rose fruité. Un collant de couleur transparent dépasse de la jupe posée bas sur les hanches. Tête chercheuse de la mode. Miuccia Prada $(\mathrm{Z})$ étonne encore [sic.] (Le Figaro, 6.3.2007).

Le choix d'effacer l'actant sémantique expérienceur $(X)$ dans l'entourage immédiat du V_émotion permet de thématiser la cause (Z) de l'émotion (l'agent causateur Miuccia Prada dans [1]. Ce cas d'ellipse implique « une désorganisation de la syntaxe usuelle » de l'énoncé (Micheli, 2010, p. 136), et engendre un « centrage discursif » particulier sur la cause de la surprise.

D'un point de vue contrastif, en arabe, les V_émotion ne semblent pas accepter de figurer dans des structures monovalentes. Cela constitue un point de divergence important entre les deux langues : en arabe, les verbes se caractérisent par l'intégration systématique au verbe d'indice de la personne qui ressent l'émotion (expérienceur X) ou celui de sa cause (Z) (Dichy, 2007).

L'actant sémantique $(Z)$ est l'élément déclencheur des émotions de la surprise ou de la colère. Il peut correspondre à une cause non-animée ou à un agent humain comme il peut se dédoubler en français. Prenons l'exemple suivant :

(2)

Mais la cohérence, dans le dédain des vedettariats, étonne toujours. Moins justifiable : le refus opposé par Gracq à toute édition bon marché de ses œuvres. "La Pléiade » : oui (1989); le poche : jamais. Fidélité fétichiste et élitiste au temps du coupe-papier, préservé par José Corti ? Nostalgie de l'effort sans quoi la lecture n'est que consommation vile et sans conséquence ? (Le Monde, 25.12.2007).

Le locuteur insiste ici sur la cause de la surprise : la cohérence. Le locuteur choisit la non-réalisation de l'expérienceur (X), ce qui permet de créer un « centrage discursif» (Fesenmeier, 2010) sur la cause de la surprise. Ce choix des arguments syntaxiques, qui constitue le « variable syntactic pivot» (Van Valin \& LaPolla, 1997), n'est pas prédictible à partir des seuls rôles sémantiques car il peut être affecté par des facteurs discursifs (p. 291). Cette non-saturation résulte d'une subjectivisation du sens relatif à la cause de l'émotion $(Z)$. Cette observation 
confirme notre hypothèse selon laquelle le choix de la réalisation ou non des actants influence la manière dont l'information s'organise au sein de la phrase.

Nous avons également relevé des emplois monovalents fréquents dans les corpus où les V_émotion sont suivis d'un SNprép :

(3)

Demi défensif infatigable pendant sa carrière où il fut surnommé « Robocop » ou "Sergent Puel », il (Z) continue à étonner par son endurance (P/Z) (Le Figaro, 17.9.2007).

Le verbe étonner est suivi du SN son endurance, introduit par la préposition par et figurant dans un complément facultatif non essentiel à la structure actancielle étudiée ici. L'Asy qui correspond à l'Asé cause $\mathrm{Z}$ se dédouble quand le locuteur choisit d'ajouter ce complément, ajout qui est mis en œuvre pour donner à une propriété du premier actant (il), d'où le recours au déterminant possessif ([son] endurance). On peut paraphraser ainsi la préposition par par : " du fait de » (Hamma, 2005) ${ }^{16}$.

Nous analysons ces ajouts (notés $\mathrm{P} / \mathrm{Z}$ ) comme un dédoublement de l'Asé cause (Z). Le locuteur fait appel à une propriété interne de l'agent (propriété inhérente à $Z$ ). Il réalise au niveau syntagmatique l'Asé cause pour atteindre une visée discursive de persuasion, et ce en insistant sur la cause de l'émotion, ce qui permet le lancement du « travail textuel d'étayage » (Jacquin \& Micheli, 2012, p. 603).

Dans la section suivante, nous nous arrêtons sur les emplois bivalents des associations verbales d'émotion en français et en arabe.

\subsubsection{Les structures bivalentes}

Les structures bivalents sont prototypiques pour les V_émotion étonner et énerver, en tant que verbes exprimant deux émotions causées. Les deux actants syntaxiques sont ainsi réalisés. Ils correspondent aux rôles sémantiques de cause (Z) et d'expérienceur $(\mathrm{X}): \mathrm{Z}$ étonnelénerve $\mathrm{X}$.

(4)

La lune de miel (Z) qu'il entretient avec l'opinion l'(X)énerve prodigieusement. (Libération, $10 / 1 / 2007)$

Du point de vue contrastif, les V_émotion sont toujours bivalents en arabe, ce qui constitue une différence avec le français (cf. section 2.1.1). En effet, cela pourrait s'expliquer par les spécificités de chaque langue : la construction des catégories grammaticales s'effectue en arabe à partir d'une racine, ce qui rend la réalisation de tous les actants indispensable au sein de la phrase :

${ }^{16}$ Hamma (2005) en étudiant les différentes valeurs sémantiques de la préposition par, aborde le degré d'agentivité supérieur inséré dans le sémantisme de cette préposition. 
(5)

Orig.ar : [... [ أغضب المشروع بن غوريون غضبا شديدا فكتب الى سفيره (Hayat97)

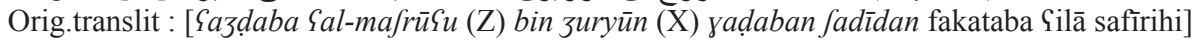
$\mathrm{V}$ _actif.passé.3masc.sing

Trad.litt. : (a-énervé le-projet $(\mathrm{Z})$ Ben Gourion $(\mathrm{X})$ colère sévère alors-il-écrit à ambassadeurde-lui $[\ldots])$

Trad.fr. : Le projet $(\mathrm{Z})$ a profondément énervé Ben Gourion $(\mathrm{X})$, il écrit à son ambassadeur [...].

Dans l'exemple ci-dessus, les Asy qui correspondent aux Asé cause (Z) et expérienceur (X) figurent tous les deux au niveau syntagmatique. C'est une structure bivalente dans laquelle le deuxième Asy correspondant à l'expérienceur (X) est « patientif» (E1 Kassas, 2005, p. 93).

Par ailleurs, des exemples de dédoublement de la cause (Z) ont été relevés dans le corpus arabe :

(6)

Orig. ar : الو اقع لقد أدهثني العرب كثير ا، بردود فعلهم على حادثة الباص (Ghad01)

Orig.translit : [ [

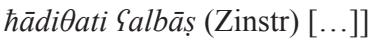

\section{V_actif.passé.3masc.sing}

Trad.litt. : le-réel a-étonné-moi (X) les-arabes (Z) dense, par-réponses actions-eux sur accident le-bus (Zinstr).

Trad.fr. : En réalité, les Arabes $(\mathrm{Z}) m(\mathrm{X})$ 'ont beaucoup étonné par leurs réactions suite à l'incident de bus (Zinstr).

La réalisation de ce dédoublement est mise en œuvre dans l'exemple ci-dessus sous la forme d'un SP avec le nom au génitif [bi-rudūdi fißli-him Yalā hādiもati Yal-bāṣ] (avec-réponses actions-eux sur accident le-bus). L'Asé cause $(Z)$ peut se dédoubler dans les structures bivalentes aussi bien en arabe qu'en français. Ce dédoublement crée une complétude sémantique : la cause $(\mathrm{Z})$, l'expérienceur $(\mathrm{X})$ et l'ajout de la cause dédoublée, notée $\mathrm{P} / \mathrm{Z}$ (propriété interne inhérente à la cause $\mathrm{Z}$ ).

Un autre mécanisme de fusion actancielle a été relevé. Observons l'exemple suivant :

(7)

James Wilson (Robert Sean Leonard), oncologue, est le meilleur (unique ?) ami de Gregory House ; celui qui le connaît bien. Ce dernier adore le pousser à bout avec des blagues de potache pour mettre à l'épreuve sa gentillesse et sa compassion $(\mathrm{P} / \mathrm{Z})$ qui l(X)'énervent tant (Le Monde, 25.2.2007).

Dans ce cas, l'Asé (P/Z) véhicule une caractéristique intrinséque de l'agent causateur, présent sur le plan syntaxique sous la forme d'un déterminant possessif (sa gentillesse et sa compassion). La structure peut être décomposée en $Z$ l'énerve tant à cause de sa gentillesse et sa compassion. Le locuteur choisit de recourir 
à ce mécanisme de fusion actancielle, qui est plus économique que le mécanisme de dédoublement décrit plus haut, et qui indique une stratégie communicative économique, moins coûteuse au niveau discursif.

Observons l'exemple suivant :

(8)

Orig. ar : أدهثت بشا بشدة إجابة الطفل الذى ألحقه أبو اه بإحدى أرقى مدارس اللغات : Shuruq)

Orig.translit : [Padhafat-hā (X) bi-fiddatin Pizābatu Pa-țtifl (P/Z) Pallad̄i Palhaqa-hu Pabawā-hu bi-\{īhā Parqā madārisi Palluzāti]

$$
\text { V_actif.passé.3fém.sing }
$$

Trad.litt. : (a-étonné-elle $(\mathrm{X})$ avec-sévérité réponse le-enfant $(\mathrm{P} / \mathrm{Z})$ qui a-joint-lui parents-lui avec-une prestigieuse écoles les-langues)

Trad.fr. : La réponse de l'enfant (P/Z), que les parents ont envoyé dans l'une des plus prestigieuses écoles de langues, $l$ '(X) a profondément étonnée.

En (8), il s'agit donc d'un mécanisme de fusion actancielle de la cause (Z).

La cause peut figurer également dans les constructions pseudo-clivées. Cellesci sont fréquentes dans le corpus français :

(9)

Ce qui énerve encore plus le maire, c'est que lui, le premier magistrat de la ville, lui qui « doit être le pilier de la prévention », n'a pas été mis au courant du déploiement des forces de l'ordre et se dit très agacé par les questions des journalistes [...] (Libération, 5.7.2007)

Le locuteur choisit, en (9), de se focaliser sur la cause de la colère, et introduit énerve encore dans une proposition relative, qui est détachée en tête de sa phrase (ce qui énerve...) et une proposition introduite par c'est. Il s'agit ici $\mathrm{d}$ l'étude du topic, d'un mécanisme de pseudo-clivage qui renvoie au thème de l'énoncé (Van Valin \& LaPolla, 1997). L'opération de « cette construction est particulièrement productive avec certains types de verbes (intéresser, aimer...) » (Roubaud, 2000, p. 9). Cette focalisation a été relevée également dans le corpus arabe. Le locuteur opère ce choix de mise en focus pour atteindre des objectifs communicatifs informatifs Creissels (2006, p. 111) au sein de la phrase, qui met à disposition " un sens complet, d'une pensée, d'un sentiment, d'une volonté » (Mauger, 1968).

Dans la section suivante, nous nous pencherons sur l'étude des V_sentiment.

\subsection{Les $\mathrm{V}$ _sentiment}

Les verbes admirer et envier font partie de la classe I des verbes psychologiques (Ruwet, 1994; Mathieu, 2000), qui expriment des sentiments interpersonnels et duratifs (admirer est de polarité positive, envier est de polarité négative). Leurs équivalents respectifs [?ązaba] et [hasada] possèdent des schèmes différents : le premier appartient 
à la forme IV (tout comme les verbes [Padhaja] (étonner) et [Gayḍaba] (énerver)), tandis que le second renvoie à la forme I (forme simple de l'actif).

\subsubsection{Les structures monovalentes}

L'examen des corpus ont permis de constater l'absence d'exemples figurant dans des constructions monovalentes avec les verbes interpersonnels admirer et envier, et ce dans les deux langues étudiées ici. Des recherches sur des sites Web ont été menées en vue de relever des cas monovalents, mais ces verbes de sentiment ne semblent pas tolérer des configurations monovalentes en supprimant le deuxième Asy objet (Y).

Nous nous pencherons, dans la prochaine sous-section, sur l'étude des structures actancielles des V_sentiment et de leurs profils discursifs, au sein des emplois actifs bivalents.

\subsubsection{Les emplois bivalents : réalisation des actants $X$ et $Y$}

Des emplois bivalents variés ont été relevés :

(10)

Le personnage du clown m'est venu spontanément, mais, rétrospectivement, je peux dire que $j^{\prime}(\mathrm{X})$ ai toujours admiré les clowns $(\mathrm{Y})$, parce qu'ils sont proches de cette très particulière ouverture d'esprit et de cœur qu'ont les enfants jusque vers 7 ans. Qu'appelez-vous « limites de la réalité »? Nous faisons comme s'il était évident que nous partagions la même réalité. C'est peut-être vrai pour les objets physiques, une table par exemple, mais dès qu'il s'agit de sentiments, de pensées, chacun d'entre nous vit séparé des autres, chacun est dans sa propre réalité comme dans une cage. Par ailleurs, même si, ou justement parce que je travaille avec le langage, je sais que c'est un outil merveilleux, mais aussi une prison. Est-ce possible d'en sortir? Quelle part de réalité pouvons-nous partager? (Libération, 27.9.2007).

En (10), la réalisation des deux actants est marquée au niveau syntagmatique : l'actant expérienceur qui éprouve le sentiment d'admiration, je (admirateur), et l'objet de son admiration, les clowns (l'être admiratif). Cela démontre que le locuteur ne peut pas opérer pour la suppression du deuxième Asy correspondant à l'Asé objet (Y), et ce en français et en arabe.

Voici un exemple issu du corpus arabe :

Orig. ar : [... [و اسمح لنا ان نحسدك قليلا وان نتعلم منأك كثير ا (Hayat97)

Orig.translit : [wa Pismah lanā Pan $\underline{n}(\mathrm{X})$-aћsuda-ka (Y) qalīlan wa Pan n-ataSallama min-ka käiran

V_actif.présent. ${ }^{\mathrm{er}}$.masc.plur

Trad.litt. : (et permets à-nous que nous(X)-envie-toi (Y) ténu et que nous-apprend de-toi dense $[\ldots])$

Trad.fr. : Et permettez-nous (X) de vous (Y) envier un peu et d'apprendre beaucoup de vous. 
$\mathrm{Au}$ niveau phrastique, le locuteur choisit en arabe $^{17}$ de remplir la place syntaxique $^{18}$ du complément du verbe [hasada] (envier), qui correspond à l'Asé objet humain animé (Y) ([-ka] (toi)).

D’autres structures trivalentes ont été aussi relevées.

\subsubsection{Les emplois trivalents}

Les verbes de sentiment peuvent réaliser trois actants dans le cadre de la phrase. Nous examinerons ces emplois dans les sous-sections suivantes.

Un cas particulier a été observé dans les deux corpus : les V_sentiment admirer et envier tolère l'ajout d'un troisième Asy qui corespond à un Asé cause : Observons l'exemple suivant :

Jean-François Deniau, disparu la semaine dernière, était « la référence » de Gisèle Chauveau, déléguée départementale de l'UDF. «Il fait partie des hommes (Y) que j'(X)ai toujours admirés pour ses combats diplomatiques $(\mathrm{Z})$, pour son courage à affronter les océans et pour sa lutte toujours positive contre la maladie » (Ouest-France, 31.1.2007).

Le locuteur choisit ici de mentionner la cause de l'admiration (Z), et ce en utilisant la construction pour $S N$ dans pour ses combats diplomatiques, pour son courage à affronter les océans et pour sa lutte toujours positive contre la maladie. Il opère ainsi pour la réalisation syntagmatique des trois actants sémantiques (X, Y et Z).

La même structure trivalente est relevée dans le corpus arabe :

Orig. ar : $[\ldots][\ldots]$

وسيتطرد عبد المقصود، الذى أحسده كثير ا على دأبه وجلده وسط كل هذه الأنو اء : (Masri2010)

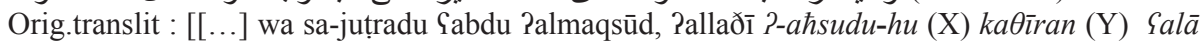
da?bi-hi wa_zaladi-hi wasața kulli hāðihi Pal-?anwā? (Z) [...]]

V_actif.présent.1 ${ }^{\text {er }}$.masc.sing

Trad.litt. : ([...] et va-été-renvoyé abdel maksud qui je-(X)-envie-lui $(\mathrm{Y})$ dense sur persévérancelui et endurance-lui milieu tout cette la-adversité (Z) [...])

Trad.fr. : Il sera renvoyé Abdel-Maksoud, que (Y) j'(X)envie beaucoup pour sa persévérance et son endurance au milieu de toute cette adversité (Z).

${ }^{17}$ Il serait fructueux de mener une étude sur les particularités de la langue arabe en tant que langue flexionnelle (plus particulièrement sur les relations grammaticales entre le radical, le suffixe et le préfixe) et sur ses spécificités morpho-syntaxiques qui peuvent ressortir des convergences en comparaison avec le français concernant la relation entre le verbe et les actants qu'il tolère (cf. El Kassas, 2005, p. 47).

${ }_{18}^{18}$ L'Asy sujet et [mafYūl bihi] (le complément du verbe) correspond à l'Asé expérienceur (X) [-n] (nous) et objet du sentiment (Y) [-ka] (toi). Il est attaché au verbe [hasada] (envier), ce qui est dû à la dépendance morphologique en arabe. 
Le verbe [?-ahsudu-] (je-envie-) est à la forme active, première personne du masculin singulier. L'Asy sujet a une fonction remplie par un pronom zéro, qui a un référent sémantique (l'expérienceur $\mathrm{X})^{19}$. L'utilisation de la préposition [Galā] (sur) suivie d'un SN : [daPbi-hi wa zaladi-hi_[...] (persévérance-lui et endurance-lui) permet au locuteur de réaliser les trois actants, ce qui lui permet de mettre en œuvre sa visée d'insistance. C'est donc la stratégie argumentative par insistance qui détermine la réalisation complète des actants (Sorba \& Novakova, 2013, p. 207).

Dans d'autres cas, le locuteur choisit de recourir à la fusion des Asé objet (Y) et cause $(Z)$ :

On a toujours admiré Frank Castorf et sa féroce intelligence, son sens du plateau, de la troupe $(\mathrm{Y} / \mathrm{Z})$. Ses acteurs sont formidables, mais le spectacle qui disloque Céline en une "guignolade» répétitive et totalement insaisissable pour ceux qui ne connaîtraient pas le texte déçoit (Le Figaro, 9.7.2007).

Dans les actants sa féroce intelligence, son sens du plateau, de la troupe marqués par la présence d'un déterminant possessif, l'objet et la cause qui provoque l'admiration $(\mathrm{Y} / \mathrm{Z})$ se fusionnent. Ces observations nous font penser à la notion de " greffe syntaxique » (Legallois, 2013) ${ }^{20}$.

L'emploi trivalent se caractérise par l'objectif « de proposer une construction discursive de la situation qui, selon le locuteur, fonde ou, au contraire, invalide l'émotion attribuée » (Micheli, 2010, p. 108). De là, découle la nécessité d'identifier les types de situations, voire les topiques (Plantin, 1997; Micheli, 2010, p. 59). C'est une « mise en fonctionnement de la langue par un acte individuel d'utilisation » (Benveniste, 1974, p. 80), qui nous permet de déceler les « actes discrets et chaque fois uniques par lesquels la langue est actualisée en parole par un locuteur » (p. 251).

\section{Conclusion}

Nous avons proposé de partir de catégories sémantiques pour la recherche des combinaisons syntaxico-sémantiques de l'affect, avec l'hypothèse que ces catégories globales pourront se retrouver dans les deux langues. Notre objectif était d'étudier la combinatoire des V_émotion dans une interface syntaxique et discrusive français-arabe. En partant de l'idée que, le locuteur choisit de réarranger ou

19 « Le pronom sujet ne possède pas d'expression morphophonologique en arabe » (El Kassas, 2012, p. 143).

${ }^{20}$ Il s'agit d'analyser la fusion de deux énoncés : par exemple, dans la manifestation s'annonce pour être exceptionnelle, on trouve la manifestation s'annonce exceptionnelle ou la manifestation est partie pour être exceptionnelle (Legallois, 2013). 
de supprimer ou de destituer les actants syntaxiques, dans le cadre de la diathèse active en fonction de ses stratégies argumentatives et de ces visées communica-

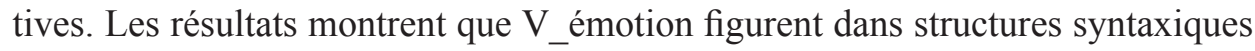
différentes des $\mathrm{V}$ _sentiment. Le fait de réaliser ou non les différents actants au niveau syntagmatique peut, en effet, influencer l'organisation de l'information dans l'énoncé, et ce dans le cadre d'une approche fonctionnelle et contrastive. Examiner le système valenciel réalisé en surface, dans le discours au sein des emplois passifs et pronominaux reste à faire.

\section{References}

Abu Ssaydeh, A. (2001). Synonymy, Collocation and the Translation. Turjuman, 10(2), 53-71.

Arbaouim N. (2010). Les dix formes de l'arabe classique à l'interface syntaxe/phonologie - Pour une déconstruction du gabarit (Doctoral disseration thesis, Université Paris 7, France).

Benveniste, E. (1974). Problèmes de linguistique générale: Vol. 2, Paris: Gallimard.

Bolly, C. (2010). Flou phraséologique, quasi-grammaticalisation et pseudo marqueurs de discours : un no man's land entre syntaxe et discours? In L. A. Johnsen, G. Cordminboeuf, \& V. Conti (Eds.), Entre syntaxe et discours. Éclairage épistémologiques et descriptions linguistiques, (pp. 11-38). Retrieved September 2, 2019, from https://journals.openedition.org/linx/1356.

Bolly, C. (2011). Phraséologie et collocations. Approche sur corpus en français L1 et L2. GRAMM-R. Études de linguistique française, 9. Bruxelles, Bern, Berlin, Frankfurt am Main, New York, Oxford, Wien: Peter Lang Verlag.

Bouchaddakh, S. (2010). Le Dico-FRAR : base de données lexicographiques bilingue françaisarabe: à paraître dans les Actes des Huitièmes journées scientifiques du réseau Lexicologie, terminologie, traduction, 15-17 octobre 2009, Lisbonne, 1-18.

Buvet P.-A., Girardin C., Gross G., \& Groud, C. (2005). Les prédicats d'affect. LIDIL, 32, 123-143. Creissels, D. (2006). Syntaxe generale. Une introduction typologique, Vol. 1 et 2. Paris: Hermes.

Dichy, J. (2007). Les compétences en traduction comme complément cognitif de l'apprentissage des langues : quelques propositions relatives à l'arabe. In Ph. Anckaert, F. El Qasem, \& J. Walravens (Eds.), Tarjama : quels fondements pour la didactique de la traduction arabe (pp. 25-34). Liège: Céfal.

El-Hasan, S. (1982). Meaning by Collocation with Illustration from Written Arabic: Ral-majzalla Pal-Sarabijja li-lYulūm Pal-\{arabijja [La revue arabe pour les sciences arabes], 2, 273-280.

El Kassas, D. (2005). Une étude contrastive de l'arabe et du français dans une perspective de génération multilingue (Doctoral dissertation thesis, Université Paris 7, France).

El Kassas, D. (2012). La catégorie de voix en arabe. Retrieved September 2, 2019, from http://www. ruslang.ru/doc/melchuk_festschrift2012/El-Kassas.pdf.

Fesenmeier, L. (2010). « Se souvenir » en français et en italien : différence(s) de centrage. In M. Iliescu, H. Siller-Runggaldier, \& P. Danler (Eds.), Actes du XXVe Congrès international de linguistique et de philologie romanes (pp. 85-96). Tübingen: Max Niemeyer.

Ghazala, H. (2007). Dictionary of Collocations English-Arabic. Beirut: Dâr al-'ilm li-l-malâyîn.

Gross, M. (1975). Méthodes en syntaxe : régime des constructions complétives. Paris: Hermann.

Halliday, M. A. K., \& Hasan, R. (1976). Cohesion in English. London: Longman.

Hamma, B. (2005). L'invariant sémantique de la préposition PAR à travers les distributions syntaxiques et sémantiques (Doctoral dissertation thesis, Université Paris X, France).

Harris, Z. S. (1988). Language and Information. New York: Columbia University Press.

Hausmann, F. J. (1989). Le dictionnaire de collocations. In F. J. Hausmann, O. Reichmann, H. E. Wiegand, \& L. Zgusta (Eds.), Wörterbücher: ein internationales Handbuch zur Lexikographie (pp. 1010-1019). Berlin, New York: De Gruyter. 
Jacquin J., \& Micheli, R. (2012). Entre texte et interaction : propositions méthodologiques pour une approche discursive de l'argumentation en sciences du langage. In F. Neveu, V. Muni Toke, P. Blumenthal, T. Klingler, P. Ligas, S. Prévost, \& S. Teston-Bonnard (Eds.), Actes du CMLF 2012 - $3^{e}$ Congrès mondial de linguistique française (pp. 599-611). Lyon: EDP Sciences.

Legallois, D. (2013). La colligation : autre nom de la collocation grammaticale ou autre logique de la relation mutuelle entre syntaxe et sémantique ? Retrieved June 28, 2019, from http://corpus. revues.org/2202.

Mathieu, Y. Y. (2000). Les verbes de sentiment : de l'analyse au traitement automatique. Paris: Éditions du CNRS.

Mauger, G. (1968). Grammaire pratique du français d'aujourd'hui : langue parlée, langue écrite. Paris: Hachette.

Mel'čuk I., Clas, A., \& Polguère A. (1999). Dictionnaire explicatif et combinatoire du français contemporain: Recherches lexico-sémantiques: Vol. 1-4. Montréal: Les Presses de l'université de Montréal.

Micheli, R. (2010). L'émotion argumentée. L'abolition de la peine de mort dans le débat parlementaire français. Paris: Cerf.

Novakova, I. (2013). Les émotions : entre lexique et discours. In A. Rabatel, A. Ferrara-Léturgie, \& A. Léturgie (Eds.), La sémantique et ses interfaces (pp. 181-204). Limoges: Lambert-Lucas.

Novakova, I., Goossens V., \& Grossmann F. (2013). Le profil actanciel et discursif des verbes de surprise et de respect. Langue française, 180, 31-46.

Novakova I., Grossmann F., \& Goossens V. (2013). Interactions entre profil discursif et structures actancielles : l'exemple des noms de surprise et de respect. In F. Baider, \& G. Cislaru (Eds.), Cartographie des émotions. Propositions linguistiques et sociolinguistiques (pp. 71-84). Paris: Presses Sorbonne nouvelle.

Plantin, Ch. (1997). L'argumentation dans l'émotion. Pratiques, 96, 81-100.

Roubaud, M.-N. (2000). Les constructions pseudo-clivées en français contemporain. Paris: Champion.

Ruwet, N. (1994). Être ou ne pas être un verbe de sentiment. Langue française, 103, 45-55.

Sorba J., \& Novakova I. (2013). Stupéfier et jalouser dans les séquences textuelles journalistiques : quel profil discursif pour quelle stratégie argumentative? Le discours et la langue. Revue de linguistique française et d'analyse du discours, 4(1), 203-220.

The Leipzig Glossing Rules: Conventions for interlinear morpheme-by-morpheme glosses: Retrieved June 2, 2019, from https://www.eva.mpg.de/lingua/pdf/Glossing-Rules.pdf.

Van Valin R., \& LaPolla R. (1997). Syntax: structure, meaning, function: Cambridge: Cambridge University Press.

Zouaidi, S. (2018). Associations sémantiques et syntaxiques: à l'exemple des combinaisons des verbes d'émotion dans les champs de la colère et de l'admiration en français et en arabe. Annales FF, 36, 197-206. DOI: 10.17951/ff.2018.36.1.197-206. 